\title{
Rise, and pronounced regional variation, in methylphenidate, amphetamine, and lisdexamfetamine distribution in the United States
}

\author{
Sneha M Vaddadi ${ }^{1}$, Nicholas J Czelatka ${ }^{1}$, Belsy Gutierrez ${ }^{1,2}$, Bhumika C Maddineni ${ }^{3,4}$, Kenneth McCall ${ }^{5}$, Brian J \\ Piper ${ }^{\text {Corresp. } 1,4}$ \\ ${ }^{1}$ Medical Education, Geisinger Commonwealth School of Medicine, Scranton, PA, United States of America \\ Biology, University of Scranton, Scranton, PA, United States \\ 3 Medicine, University of Texas Southwestern Medical Center, Dallas, Texas, United States of America \\ ${ }^{4}$ Geisinger Precision Health Center, Center for Pharmacy Innovation and Outcomes, Forty Fort, PA, United States of America \\ 5 Pharmacy, University of New England, Portland, ME, United States \\ Corresponding Author: Brian J Piper \\ Email address: bpiper@som.geisinger.edu
}

Background. The prescription stimulants methylphenidate, amphetamine, and lisdexmafetamine are sympathomimetic drugs with therapeutic use. They are designated in the United States as Schedule II substances, defined by the 1970 Controlled Substances Act as having a "high potential for abuse". Changing criteria for the diagnosis of Attention Deficit Hyperactivity Disorder in 2013 and the approval of lisdexamfetamine for binge eating disorder in 2015 may have impacted usage patterns. This report compared the pharmacoepidemiology of these stimulants in the United States from 2010-2017.

Methods. Distribution of amphetamine, methylphenidate, lisdexafetamine were examined via weights extracted from the Drug Enforcement Administration's (DEA) Automated Reports and Consolidated Ordering System (ARCOS). Median stimulant daily dosage per patient was determined for a regional analysis. The percent of cost and prescriptions attributable to each stimulant and atomoxetine in Medicaid from the "Drug Utilization 2018 - National Total" from the Centers for Medicare and Medicaid was determined.

Results. There was a rise in amphetamine (+67.5\%) and lisdexamfetamine (+76.7\%) from 2010-2017. The change in methylphenidate $(-3.0 \%)$ was modest. Persons/day stimulant usage was lower in the West than in other US regions from 2014-2017. There was a negative correlation $(r(48)=-0.43$ to $-0.65, p<$ .05) between the percent Hispanic population per state and the daily dose/population per stimulant. Methylphenidate formulations accounted for over half $(51.7 \%)$ of the $\$ 3.8$ billion reimbursed by Medicaid and the plurality (45.4\%) of the $\mathbf{2 2 . 0}$ million prescriptions. Amphetamine was responsible for less than one-fifth (18.4\%) of cost but one-third of prescriptions (33.6\%). Lisdexamfetamine's cost $(26.0 \%)$ exceeded prescriptions (16.3\%).

Conclusion: The rising amphetamine and lisdexamfetamine distribution may correspond with a rise in adult ADHD diagnoses. Regional analysis indicates that stimulant distribution in the West may be distinct from that in other regions. The lower stimulant distribution in areas with greater Hispanic populations may warrant further study. 
1

\section{Rise, and Pronounced Regional Variation, in}

\section{Methylphenidate, Amphetamine, and Lisdexamfetamine}

\section{Distribution in the United States}

Sneha M. Vaddadi ${ }^{1}$, Nicholas J. Czelatka ${ }^{1}$, Belsy D. Gutierrez ${ }^{1,2}$, Bhumika C. Maddineni ${ }^{3,4}$,

Kenneth L. McCall ${ }^{5}$, Brian J. Piper ${ }^{1,4}$

${ }^{1}$ Geisinger Commonwealth School of Medicine, Scranton, PA, USA

${ }^{2}$ University of Scranton, Scranton, PA, USA

${ }^{3}$ University of Texas Southwestern Medical Center, Dallas, TX, USA

${ }^{4}$ Center for Pharmacy Innovation and Outcomes, Forty Fort, PA, USA

${ }^{5}$ University of New England, Portland, ME, USA

Corresponding Author:

Brian J. Piper

Department of Medical Education

Geisinger Commonwealth School of Medicine

Scranton, PA 18409, USA

Email address: bpiper@som.geisinger.edu; psy391@gmail.com

\section{Abstract}


32 Background. The prescription stimulants methylphenidate, amphetamine, and lisdexmafetamine

33 are sympathomimetic drugs with therapeutic use. They are designated in the United States as

34 Schedule II substances, defined by the 1970 Controlled Substances Act as having a "high

35 potential for abuse”. Changing criteria for the diagnosis of Attention Deficit Hyperactivity

36 Disorder in 2013 and the approval of lisdexamfetamine for binge eating disorder in 2015 may

37 have impacted usage patterns. This report compared the pharmacoepidemiology of these

38 stimulants in the United States from 2010-2017.

39 Methods. Distribution of amphetamine, methylphenidate, lisdexafetamine were examined via

40 weights extracted from the Drug Enforcement Administration’s (DEA) Automated Reports and

41 Consolidated Ordering System (ARCOS). Median stimulant daily dosage per patient was

42 determined for a regional analysis. The percent of cost and prescriptions attributable to each

43 stimulant and atomoxetine in Medicaid from the "Drug Utilization 2018 - National Total" from

44 the Centers for Medicare and Medicaid was determined.

45 Results. There was a rise in amphetamine (+67.5\%) and lisdexamfetamine (+76.7\%) from 2010-

46 2017. The change in methylphenidate (-3.0\%) was modest. Persons/day stimulant usage was

47 lower in the West than in other US regions from 2014-2017. There was a negative correlation

$48(r(48)=-0.43$ to $-0.65, p<.05)$ between the percent Hispanic population per state and the daily

49 dose/population per stimulant. Methylphenidate formulations accounted for over half (51.7\%) of

50 the $\$ 3.8$ billion reimbursed by Medicaid and the plurality (45.4\%) of the 22.0 million

51 prescriptions. Amphetamine was responsible for less than one-fifth (18.4\%) of cost but one-third

52 of prescriptions (33.6\%). Lisdexamfetamine’s cost (26.0\%) exceeded prescriptions (16.3\%).

53 Conclusion: The rising amphetamine and lisdexamfetamine distribution may correspond with a

54 rise in adult ADHD diagnoses. Regional analysis indicates that stimulant distribution in the West

55 may be distinct from that in other regions. The lower stimulant distribution in areas with greater

56 Hispanic populations may warrant further study. 


\section{Introduction}

58 Stimulants are sympathomimetic substances that mimic the effects of the sympathetic

59 nervous system and have Food and Drug Administration approved indications (Coghill et al.

60 2014; King, Casavant, Spiller, Hodges, et al. 2018). Methylphenidate, amphetamine, and other

61 stimulants are widely used in the US for Attention Deficit Hyperactivity Disorder (ADHD), a

62 psychiatric disorder characterized by hyperactivity, inattention, executive function deficits and

63 emotional dysregulation (Bădescu et al., 2016; Subcommittee on Attention-Deficit/Hyperactivity

64 Disorder \& Steering Committee on Quality Improvement and Management, 2011). ADHD is one

65 of the more common biopsychosocial disorders, with an estimated national prevalence of $9.4 \%$,

66 or 6.1 million, in children from age 2-17 in 2016 per the National Survey of Children's Health.

67 Of those currently diagnosed with ADHD, over three-fifths (62.0\%) were taking medication,

68 accounting for $5.1 \%$ of the children from age 2-17 in the US (Danielson et al. 2018). With the

69 implementation of the Patient Protection and Affordable Care Act in 2014 and the transition to a

70 "fee-for-service" program in 39 states by 2016, Medicaid is the largest payer for mental health

71 services in the US. Medicaid is a federal and state program that assists in healthcare costs for

72 patients with limited resources, and provides insurance to over 36 million children as of June

73 2020, offering coverage to a sizable portion of patients on stimulant medications (Chorniy,

74 Currie, \& Sonchak 2018). Amphetamine-type stimulants accounted for the highest proportion of

75 medication expenditures for Medicaid enrolled children (Cohen, Hall, M., Lopert, et al. 2017). To

76 date, the twelve states that have not elected to expand Medicaid are located primarily in the south

77 and midwest (Kaiser Family Foundation, 2021). Despite the growth in ADHD, there is variation

78 within cultural communities. Although one of the largest ethnic minorities in the United States,

79 Hispanic youths have reduced ADHD diagnosis and stimulant use (Pennap Burcu, Safer et al.,

80 2017; Velasco-Mondragon et al., 2016). 
82 criterion released in 2013 in the Diagnostic and Statistical Manual (DSM) 5, ADHD diagnostic

83 criteria has been more inclusive of adolescents and adults. As of 2018, approximately 4\% of US

84 adults are afflicted with ADHD and two-thirds of children continue to experience at least one

85 ADHD symptom throughout their lives (Wei et al., 2018). Lisdexamfetamine and mixed

86 amphetamine salts were found to cause a significant improvement in adult ADHD symptoms

87 without symptoms rebound after ceasing medication (Buoli, Serati, \& Cahn, 2016; Stevens,

88 Wilens \& Stern, 2013). In contrast, two Cochrane reviews concluded the quality of evidence base

89 assessing the efficacy of amphetamine for child, adolescent, and adult ADHD was "low to very

90 low” (Castells, Blanco-Silvente, Cunill, 2015; Punja et al. 2016). Stimulants have also been

91 approved for other medical conditions, such as the approval of lisdexamfetamine for binge eating

92 disorder in 2015 (Guerdkikova et al. 2016).

93

Stimulant use has been associated with mild adverse effects such as appetite and sleep

94 disturbances that impact quality of life, modest reductions in height in children and adolescents,

95 and small elevations in blood pressure and heart rate but the long-term adverse effects of these

96 substances are not well established (Coghill et al. 2014; Groenman et al., 2017). A recent study

97 determined that patients prescribed stimulants had a nine-fold elevated risk of developing basal

98 ganglia and cerebellar disorders, and it is suggested by the authors that this may be indicative of

99 the ADHD phenotype as increased risk of these motor disorders (Curtin et al. 2018).

100 Interestingly, a history of recreational methamphetamine or amphetamine misuse was associated

101 with a three-fold elevated risk of developing Parkinson's (Curtin et al. 2015). A meta-analysis of

102 cross-sectional Positron Emission Tomography investigations showed that long-term blockade of

103 the dopamine transporter with ADHD pharmacotherapies caused neuroadaptive striatal elevations

104 in this protein (Fusar-Poli et al. 2012) which was subsequently confirmed in a longitudinal report

105 (Wang et al. 2016). 
107 the US Drug Enforcement Administration (DEA) as Schedule II drugs, which are defined as 108 those with a "high potential for abuse, with use potentially leading to severe psychological or

109 physical dependence.” (DEA, 2021). Methylphenidate had slightly higher affinity for the

110 dopamine and norepinephrine transporters than cocaine (Dan \& Gu, 2004). Methylphenidate, D-

111 amphetamine, and cocaine usually share discriminative stimulus effects (Kollins, MacDonald, \&

112 Rush, 2001). The prodrug lisdexamfetamine may be resistant to misuse but that does not prevent

113 consuming multiple doses orally. Several data sources have produced evidence indicating there is

114 appreciable non-medical use of prescription stimulants. The Monitoring the Future survey of

115 recreational drug use determined that $4.6 \%$ of $12^{\text {th }}$ graders misused Adderall (amphetamine) in

1162018 (National Institute on Drug Abuse, 2018). Five million people misused a prescription

117 stimulant in the US in the past year (Substance Abuse and Mental Health Services

118 Administration, 2019). Use and misuse may be particularly high among some populations.

119 In one institution in Puerto Rico, half of medical students reported a history with prescription

120 stimulants and $89.4 \%$ of this subset used these agents without a prescription (Acosta et al. 2019).

121 Calls to US poison control centers between 2007 and $2012(\mathrm{~N}=23,533)$ were more likely to

122 involve amphetamine than the pro-drug lisdexamfetamine (Kandland \& Klein Schwartz, 2015).

123 Exposures involving ADHD medications $(\mathrm{N}=156,635$, i.e one call to poison control every fifty

124 minutes) for patients age $\leq 19$ increased by 71.2\% from 2000 to 2011 (King, et al. 2018). The

125 number of population corrected exposures was $42.0 \%$ lower in the western relative to the

126 midwestern states (King et al. 2018).

127 With increasing prevalence of medicated ADHD in children and adults as well as

128 lisdexamfetamine in adults with binge eating disorder, there is a greater need to understand the

129 extent of stimulant use nationally. This report utilized the US DEA's Automated Reports and

130 Consolidated Ordering Systems (ARCOS) comprehensive database to evaluate changes in use of 
131 amphetamine, methylphenidate, and lisdexamfetamine nationally from prior years to 2017. We

132 extended upon past research (Piper et al., 2018a) by investigating the overall change of stimulant 133 use from 2010-2017. We then calculated daily dose values to investigate the change in use from

1342016 and 2017. We also explored variations in use in the Hispanic population and geographical

135 regions (King et al. 2018). Finally, we explored stimulant use and expenditures within Medicaid 136 via Medicaid reimbursements to healthcare facilities. 


\section{Materials \& Methods}

138 Data Sources

Stimulant data was extracted from the DEA's ARCOS, a national database containing a

140 yearly updated report of retail drug distribution from manufacturers and distributors. Extracted

141 data included total grams of stimulant use (two-hundred $5 \mathrm{mg}$ pills $=1 \mathrm{~g}$ ) per drug per state (50

142 states excluding US territories) from 2010 to 2017. Three Schedule II stimulants were examined:

143 amphetamine, methylphenidate and lisdexamfetamine. This database has been frequently used in

144 prior pharmacoepidemiology reports (Atluri, Sudarshan, \& Manchikanti, 2014; Bokhari et al.

145 2005; Collins et al. 2019; Davis et al. 2020; Pashmineh et al. 2020; Piper et al. 2020; Simpson et

146 al. 2019). The main unit of data reported of drug by weight may be less familiar than other units

147 like the number of prescriptions. ARCOS was validated by examining the total weight of

148 oxycodone in this database relative to that reported in a Prescription Monitoring Program which

149 revealed a high correlation $(r=0.99)$ (Piper et al. 2018b). Further, a comparison of stimulant use

150 by weight by zip code in a high versus low classification indicated an excellent concordance

151 (96.5\%) between ARCOS and California's Prescription Drug Monitoring Program (Bokhari et al. 152 2005).

153 Our goal was to examine the change in stimulant use and in number of patients utilizing

154 Schedule II stimulants, but ARCOS data is limited to the total quantity of Schedule II stimulants

155 distributed in a geographical location. To approximate the changes in number of patients utilizing

156 these substances, we calculated the median estimated daily dose per person (mg/person/day) for

157 each stimulant. These values were calculated from 2018 de-identified data $(\mathrm{N}=88,202)$ from the

158 electronic health record (EHR) of the Geisinger Health System, an integrated health delivery

159 system in central and northeastern Pennsylvania. The calculated values, termed "daily dosage”,

160 are $20 \mathrm{mg} /$ day/person for methylphenidate and amphetamine and $40 \mathrm{mg} / \mathrm{day} / \mathrm{person}$ for

161 lisdexamfetamine based on the median dose prescribed to patients. These values were then used 
162 for determination of the change in daily doses in 2016-2017 and regional comparison by dividing

163 total grams extracted from ARCOS by daily dosage data (e.g. methylphenidate $=20 \mathrm{mg}$ ). An

164 analysis was completed with state-specific Hispanic population data. The percent Hispanic

165 population per state was obtained from the demographic profiles from the Pew Research Center

166 (Pew Research Center, 2021).Trends of stimulant use in the Medicaid system was investigated

167 using data from the "Drug Utilization 2018 - National Total” from the Centers for Medicare and

168 Medicaid (Centers for Medicare and Medicaid Services, 2021). This form was used to extract

169 number of prescriptions and total cost of substances that were reimbursed to prescribing

170 healthcare facilities by Medicaid for methylphenidate, lisdexamfetamine, and amphetamine.

171 Atomoxetine (non-scheduled and therefore not reported in ARCOS) was also obtained. IBM

172 Micromedex was used to obtain formulation names. Institutional Review Board approval was

173 obtained from the University of New England (\#20180410-009) and Geisinger (2019-0598).

174

175 Data Analysis

176 Total stimulant use from 2010-2017 was investigated by averaging extracted stimulant

177 weight (grams) per state and comparing per year for each stimulant. Data was deemed to be

178 significant $(\mathrm{p}<0.05)$ after a paired t-test. This provided an overall index of the temporal profile.

179 For further investigation into change from 2016-2017, ARCOS aggregate data was divided by

180 median daily dosage values (mg/person/day). These values were divided by population per state

181 and labeled “Daily Dose/population”. Conversion to daily doses provides units that some may

182 find more intuitive than total weights. The percent change in these values per state per drug from

183 2016-2017 were calculated and compared to provide recent changes and extend upon our prior

184 report (Piper et al. 2018). Values were deemed significant as $\geq 1.96$ standard deviations above

185 and below the mean, accounting for values outside a 95\% confidence interval. A heat map was

186 constructed with Excel using the percent data detailed above, and with the values from 2017. 
187 Regional variance analysis was conducted by dividing ARCOS aggregate data per US region

188 (Midwest, Northeast, South, West) and dividing by daily dosage. These values were labeled 189 “Person/day” and compared for all three stimulants from 2014-2017. Data was deemed to be 190 significant $(\mathrm{p}<0.05)$ after an unpaired t-test. For all above calculations, outliers were determined

191 through a Grubbs analysis and significant values were excluded. A correlational analysis was 192 done with daily dose/population values and Hispanic population data per state in 2016 and 2017.

193 This was completed to verify the utility of the daily dose measure and to extend upon earlier

194 findings (Piper et al. 2018a). The amount of Medicaid spending (\$) and number of prescriptions

195 in 2018 for each Schedule II stimulant and atomoxetine were extracted and each presented as

196 percentage of a whole. Health care spending in the US was $17.7 \%$ of the nation's gross domestic

197 product (Centers for Medicare and Medicaid Services, 2021) so characterizing sources of

198 spending for common pharmacotherapies is an important concern. Identifying the significance of 199 spending in a major public program such as Medicaid, utilized by millions of children in the 200 United States, will further justify the need for investigation in stimulant use. Alpha values 201 obtained that were below the standard $(\mathrm{p}<.05)$ threshold were noted. Variance was reported as 202 the standard error of the mean (SEM).

\section{Results}

Stimulant use increased $+67.5 \%$ for amphetamine and $+76.7 \%$ for lisdexamfetamine from 2010-2017 on average across all fifty states. In contrast, methylphenidate use decreased slightly

208 (-3.0\%). For amphetamine and lisdexamfetamine, there was a significant increase in the total 209 stimulant use compared to 2010 starting from 2014 (Figure 1A). Further investigation into the 210 change from 2016-2017 was completed with a daily-dose and population-corrected analysis. The

211 percent change in daily dose/population across fifty states (with New Mexico excluded as an 212 outlier) was $+4.6 \%$ for amphetamine, $+2.3 \%$ for lisdexamfetamine, and $-1.4 \%$ for 
213 methylphenidate (Figure 1B, C). The preponderance (85.0\%) of states increased their

214 amphetamine and over two-thirds (72.0\%) increased their lisdexamfetamine use. In contrast,

$21586.0 \%$ of states decreased their methylphenidate use. Wisconsin, South Dakota and West Virginia

216 were all significantly greater than the mean for amphetamine. Hawaii had a significantly greater

217 value while Nevada and South Dakota had lower values compared to the national average for

218 methylphenidate. For lisdexamfetamine, South Dakota had a significantly greater value while

219 Wisconsin had a lower value relative to the mean (Figure 1D).

220 The daily dose/population values for 2017 are depicted in the heat map in Figure 2A,

221 indicating pronounced regional variance. There was a six-fold difference between values for the

222 highest (12.2) and lowest (2.9) states. Six states with the lowest values of daily dose/population

223 were all in the Western region of the United States. A regional analysis was completed to further

224 investigate geographic-based variability. A comparison of persons/day per region from 2014-

2252017 revealed a significant difference in the values for the Midwest, Northeast, and South

226 compared to the West consistently from 2014-2017 (Figure 2B). Analysis with the percent

227 Hispanic population and Daily dose/population identified a negative correlation for each

228 stimulant in 2016. These findings were replicated for 2017 (Figure 3). States with a greater

229 portion of Hispanic populations had lower stimulant distributions.

230 The Medicaid program spent \$3,765,776,679 for 22,024,008 prescriptions for

231 methylphenidate, lisdexafetamine, amphetamine and atomoxetine in 2018. Methylphenidate

232 accounted for about half of cost (51.7\%) and prescriptions (45.4\%). Amphetamine was

233 responsible for less cost (18.4\%) than prescriptions (33.6\%). Lisdexamfetamine’s cost (26.0\%)

234 exceeded prescriptions (16.3\%). Further analysis was completed examining the breakdown of

235 different formulations (Table 1).

236

237

Discussion 
239 prior years to 2017, and indicated an overall increase. An analysis for total grams from 2010-

2402017 and a daily dose and population-considered analysis from 2016-2017 indicated a rise in

241 amphetamine and lisdexamfetamine and no appreciable change in methylphenidate.

242 This pattern of stimulant use change may be at least partially explained by an increase in

243 adult ADHD diagnoses and treatment (Bădescu et al, 2016). The revision of the ADHD criteria in

244 the DSM-5 was more inclusive of adult ADHD, leading to more adults meeting the requirements

245 for diagnosis than that for the DSM-IV (Epstein \& Loren, 2013). A 2018 study looking at ADHD

246 treatment in privately-insured women aged 15-44 found a similar pattern in these three stimulants

247 as this study, with the largest change in stimulant use being in the age range of 25-29 years

248 (Anderson, Ailes, Danielson, et al., 2018). Studies have also indicated variance in drug efficacy

249 for long term treatment in adult ADHD. Though methylphenidate is considered a first-line

250 treatment for child and adolescent ADHD, the long-term ( $>12$ months) efficacy of stimulants is

251 not well established (Punja et al 2016). Alternate uses for stimulants outside of AHDH treatment

252 may also contribute to these patterns in stimulant use. Lisdexamfetamine is a well-tolerated

253 treatment for moderate to severe binge eating disorder (Heo \& Duggan, 2017). Amphetamine and

254 methylphenidate are also employed in the treatment of apathy in Alzheimer's patients and other

255 neuropsychiatric conditions in the elderly (Dolder, Davis, \& McKinsey, 2010). Further

256 exploration is needed on how the expansion of stimulant use in other neuropsychiatric conditions

257 like binge eating disorder (Heo \& Duggan, 2017) impacts the use of stimulants.

258 The percent Hispanic population had a negative correlation with stimulant use per state for

2592016 and 2017. Other studies have also indicated a lower stimulant use by Hispanic children

260 compared to their non-Hispanic peers (Davis, Feygin, Creel, et al., 2019). Young Hispanic adults

261 and children have a significantly lower use of outpatient mental health services for mental health

262 and substance abuse care (Marrast, Himmelstein, \& Woolhandler, 2016). This correlation of 
263 lower stimulant use in states with greater Hispanic population may indicate a lower use of

264 stimulants among the Hispanic population. This may be attributed to difficulties with access to

265 healthcare, as prior to the implementation to the Affordable Care Act (ACA) in 2014, 30\% of

266 Hispanics reported no health insurance compared to $11 \%$ of non-Hispanic whites (Velasco-

267 Mondragon, Jimenez, Palladino-Davis, et al., 2016). Along with social factors such as language

268 barriers, cultural factors such as a perceived difference in the need for outpatient mental health

269 care may also explain differences in resource utilization (Alegría, Canino, Rios, et al, 2002). The

270 cumulative effect of these sociocultural or pharmacoeconomic factors may lead to individuals

271 being unable or hesitant to seek medical attention for ADHD symptoms.

272 Our regional analysis with data controlled for daily dosage found that the West has a

273 significantly lower schedule II stimulant use compared to the South, Northeast, and Midwest.

274 This pattern was seen in other studies spanning from 1998-2018 focusing on both child and adult

275 ADHD, with the West having the lowest ADHD prevalence or change in stimulant use (Huber,

276 Kim, Kim, et al., 2018; Piper et al., 2018a; Xu, Strathearn, Liu, et al., 2018). The availability of

277 specialty health care providers in the US exhibits pronounced regional differences. One study

278 indicated a nine-fold difference of psychiatrists per one-hundred thousand state population

279 between the highest and lowest states (Beck, Page, Buche et al. 2018). The west contained half of

280 the ten states with the fewest psychiatrists while the northeast area contained nine of ten states

281 with the most psychiatrists (Beck, Page, Buche et al. 2018). An earlier ARCOS report determined

282 that counties with greater stimulant use had more physicians per capita and were more affluent

283 (Bokhari et al. 2005). Calls to poison control centers involving ADHD medications were lower in

284 the West relative to the South and Midwest (King et al. 2018). Though frequently reported, this

285 pattern has little explanation and may be due to various factors. A 2015 report suggests that with

286 many states of higher altitude located primarily in the West, the altitude may serve as a protective

287 factor against ADHD by increasing dopamine levels (Huber et al., 2018). However, others are 
288 skeptical of the altitude hypothesis for lower ADHD levels in the Rocky Mountain states (Arns,

289 Swanson, Arnold, 2015). As noted above, cultural diversity may also play a role. Of the youth in

290 California, almost 40\% are Hispanic, an ethnicity that has significantly lower stimulant use

291 (Pennap et al., 2017). Other factors may also contribute to these regional differences including

292 pharmacy policies, Medicaid policies, rates of uninsured, or state laws regarding use of

293 psychiatric medications (Fulton, Scheffler, \& Hinshaw, 2015).

294 It was also noteworthy that secondary analyses within Medicaid identified much greater use

295 of methylphenidate than lisdexamfetamine and other stimulants which was unlike that observed

296 with ARCOS. Prior Medicaid research has examined stimulants as a group without differentiation

297 of individual agents (Cummings et al. 2017; Ji et al. 2018; Raghavan et al. 2012). It is notable

298 that twenty-seven states in 2015 had enacted Medicaid policies to be congruent with the 2011

299 American Academy of Pediatrics guidelines that clinicians refer parents of preschoolers (age 4-5)

300 for training in non-pharmacological behavior therapy and subsequently only treat with

301 medication if the behavioral treatment failed to sufficiently improve functioning (Wolraich et al.

302 2011). Seven states (AZ, FL, IL, LA, MA, VA, and WV) required use of non-medication before

303 medication (Hulkower et al. 2017). The pattern of prescriptions, and expenditures by formulation

304 among Medicaid recipients warrants further attention in updates of this study.

305 Despite stimulants being classified as schedule II substances due to their potential for abuse,

306 national surveys of adolescents and young-adults indicate that a subset of prescribed stimulants

307 are used for non-medical purposes (NIDA, 2019) or may result in calls to poison control centers

308 (King et al. 2018). In addition to misuse potential, another concern with the expanding use is the

309 potential of drug-drug interactions with many medications, including MAO oxidase inhibitors,

310 vasopressors, and coumadin anticoagulants (Groenman et al., 2017; Subcommittee on Attention-

311 Deficit/Hyperactivity Disorder \& Steering Committee on Quality Improvement and Management,

312 2011). Based on total amount reimbursed, Medicaid data indicates that approximately 50\% of the 
313 expense is due to methylphenidate use, while lisdexamfetamine expense was approximately $25 \%$.

314 Stimulants accounted for over one-fifth of the outpatient medication expenditure by Medicaid for

315 children (Cohen et al. 2017) and supports the need for further investigation into stimulant use.

316 Ongoing discussions among the one-fifth of the US states that have not yet expanded Medicaid

317 (e.g. Georgia, Florida, North Carolina, Wisconsin) (Kaiser Family Foundation, 2021) may

318 encourage increased attention on pharmacoeconomics including coverage for cognitive

319 enhancing agents like atomoxetine with the least misuse potential. Stimulant use in ADHD

320 treatment is generally regarded as safe and efficacious when used as directed with well over a

321 half-century history (Coghill et al. 2014; Cortese et al. 2018; Rasmussen, 2015). However, some

322 studies indicate controversial safety and efficacy of these schedule II stimulants, which is of

323 concern with the preponderance of methylphenidate use and the increase in lisdexamfetamine

324 (Castells et al. 2015; Cortese, Adamo, Del Giovane, et al. 2018; Punja et al. 2016; Storebo,

325 Ramstad, Krogh, et al. 2015).. This increasingly ubiquitous stimulant distribution and use

326 indicates the need for further investigation.

327 There are several limitations to this pharmacoepidemiological study. Although ARCOS is

328 inclusive of all Schedule II stimulant use, and Medicaid covers an important subset of the US

329 population, one limitation is the use of percent Hispanic population per state as determined by the

330 US Census for correlations with stimulant use. Analysis via zip code (Bokari et al. 2005) or a

331 more focused geographical delineation or using EMR may provide more conclusive associations.

332 Another caveat is the use of Medicaid reimbursement for depicting stimulant trend within the

333 expansive population covered under this public program. The predominance of certain stimulants

334 may be impacted by variations in what formulations are reimbursed rather than reflecting the true

335 demand for each stimulant. Future investigations could examine the contribution of Medicaid

336 policies or include other data sources to characterize the populations with the most pronounced 
337 changes in stimulants (i.e. adults with binge eating disorder versus ADHD, preschoolers,

338 pregnant women, or dementia patients).

340 Conclusions

341 In conclusion, this report identified increases in distribution in amphetamine and

342 lisdexamfetamine in the United States using DEA data. Examination of Medicaid revealed \$3.8

343 billion USD in spending for stimulant medications in 2018. Further investigation is needed to

344 better understand the sociocultural or economic factors mediating the pronounced regional and

345 cultural variance observed. Increased pharmacoecconomic investigations may be warranted for

346 this ubiquitous class of medications.

347

348

349

350

351

352

353

354

355

356

357

358

359

360

361

362

363

364

365

\section{Acknowledgements}

The generation of heat maps was done with the help of Daniel Kaufman, MS. This project was

also completed with the technical assistance of Iris Johnston. Publication costs were generously provided by the Geisinger Commonwealth School of Medicine.

\section{References}

Acosta, D. L., Fair, C. N., Gonzalez, C. M., Iglesias, M., Maldonado, N., Schenkman, N., Valle, S., Velez, J., Mejia, L. (2019). Nonmedical use of d-amphetamines and methylphenidate in medical students. Puerto Rico Health Science Journal 38(3):185-188.

Alergria, M., Canino, G., Rios, R., Vera, M., Calderon, J., Rusch, D., \& Ortega, A. N. (2002). Inequalities in use of specialty mental health services among Latinos, African Americans, and non-White Latinos. Psychiatric Services, 53, 1547-1555. doi: 10.1176/appi.ps.53.12.1547

Anderson, K. N., Ailes, E. C., Danielson, M., Lind, J. N., Farr, S. L., Broussard, C. S., \& Tinker, S. C. (2018). Attention-Deficit/Hyperactivity Disorder medication prescription claims among privately insured women aged 15-44 Years - United States, 2003-2015. Morbidity and Mortality Weekly Report, 67(2), 66. 
366

367

368

369

370

371

372

373

374

375

376

377

378

379

380

381

382

383

384

385

386

387

388

389

390

391

392

393

394

395

396

397

398

399

Arns, M., Swanson, J. M., Arnold, L. E. (2018). ADHD prevalence: Altitude or sunlight? Better understanding of the interrelations of dopamine and the circadian system. Journal of Attention Disorders 22:163-166.

Atluri, S., Sudarshan, G., Manchikanti, L. (2014). Assessment of the trends in medical use and misuse of opioid analgesics from 2004 to 2011. Pain Physician. 17(2): E119-28.

Bădescu, G. M., Filfan, M., Sandu, R. E., Surugiu, R., Ciobanu, O., \& Popa-Wagner, A. (2016). Molecular mechanisms underlying neurodevelopmental disorders, ADHD and autism. Romanian Journal of Morphology and Embryology, 57(2), 361-366.

Beck, A. J., Page, C., Buche, J., Rittman, D., Gaiser, M. (2018). Estimating the distribution of the U.S. psychiatric subspecialist workforce. University of Michigan Behavioral Health Workforce Research Center, Accessed 2/21/2021 at: https://www.behavioralhealthworkforce.org/wp-content/uploads/2019/02/Y3-FA2-P2Psych-Sub Full-Report-FINAL2.19.2019.pdf

Bokhari, F., Mayes, R., Scheffler, R. M. (2005). An analysis of the significant variation in psychostimulant use across the U.S. Pharmacoepidemiology \& Drug Safety, 14, 267-75. doi: $10.1002 / p d s .980$.

Buoli, M., Serati, M., \& Cahn, W. (2016). Alternative pharmacological strategies for adult ADHD treatment: a systematic review. Expert Review of Neurotherapeutics, 16(2), 131144. doi: 10.1586/14737175.2016.1135735.

Castells, X., Blanco-Silvente, L., Cunill R. (2018). Amphetamines for Attention Deficit Hyperactivity Disorder (ADHD) in adults. Cochrane Database Systematic Reviews 8(8):CD007813. doi: 10.1002/14651858.CD007813.pub3.

Centers for Medicare and Medicaid Services. (2021). Accessed 2/21/2021 at: https://www.cms.gov/Research-Statistics-Data-and-Systems/Statistics-Trends-andReports/NationalHealthExpendData/NationalHealthAccountsHistorical\#: :text=U.S. \%20health\%20care\%20spending\%20grew,For\%20additional\%20information\%2C\%20see $\% 20$ below.

Chorniy, A., Currie, J., \& Sonchak, L. (2018). Exploding asthma and ADHD caseloads: The role of Medicaid managed care. Journal of Health Economics, 60, 1-15. doi: 10.3386/w23983

Coghill, D.R., Caballero, B., Sorooshian, S., Civil, R. (2014). A systematic review of the safety of lisdexamfetamine. CNS Drugs, 28, 497-511. doi: 10.1007/s40263-014-0166-2.

Cohen, E., Hall, M., Lopert, R., Bruen, B., Chamberiain, L. J. Bardach, N., Gedney, J., Zima, B.T., Chamberlain, L.J. \& Berry, J.G., (2017). High-expenditure pharmaceutical use among children in Medicaid. Pediatrics, 140:e20171095. doi: 10.1542/peds.2017-1095.

Peer) reviewing PDF | (2020:10:53393:2:1:NEW 3 Nov 2021) 
400 Collins, L. K., Pande, L. J., Chung, D. Y., Nichols, S. D., McCall, K. L., Piper, B. J. (2019).

$401 \quad$ Trends in the medical supply of fentanyl and fentanyl analogues: United States, 2006 to

402 2017. Prev Med. 123:95-100. doi: 10.1016/j.ypmed.2019.02.017.

403 Cortese, S., Adamo, N., Del Giovane, C., Mohr-Jensen, C., Hayes, A. J., Carucci, S., Atkinson,

404 L. Z., Tessari, L., Banaschewski, T., Coghill, D., Hollis, C., Simonoff, E., Zuddas, A,

405 Barbui, C., Purgato, M., Steinhausen, H.C., Shokranen, F., Xia J., \& Cipriani, A. (2018).

406 Comparative efficacy and tolerability of medications for attention-deficit hyperactivity

407 disorder in children, adolescents, and adults: a systematic review and network meta-

$408 \quad$ analysis. Lancet Psychiatry, 5(9), 727-738.

409 Cummings, J. R., Ji, X., Allen, L., Lally, C., Druss, B. G. (2017). Racial and ethnic differences in

410 ADHD treatment quality among Medicaid-enrolled youth. Pediatrics, 139(6), e20162444.

411 doi: 10.1542/peds.2016-2444.

412 Curtin, K., Fleckenstein, A. E., Keeshin, B. R., Yurgelun-Todd, D. A., Renshaw, P. F., Smith, K.

413 R., and Hanson, G.R. (2018). Increased risk of diseases of the basal ganglia and

414 cerebellum in patients with a history of attention-deficit/hyperactivity disorder.

415 Neuropsychopharmacology 43, 2548-2555. doi: 10.1038/s41386-018-0207-5.

416 Curtin, K., Fleckenstein, A.E.,Robison, R. J., Crookston, M. J., Smith, K. R., Hanson, G. R.

417 (2015). Methamphetamine/amphetamine abuse and risk of Parkinson's disease in Utah: A

418 population based assessment. Drug \& Alcohol Dependence 146, 30-38.

419 doi:10.1016/j.drugalcdep.2014.10.027.

420 Danielson, M. L., Bitsko, R. H., Ghandour, R. M., Holbrook, J. R., Kogan, M. D., \& Blumberg,

421 S. J. (2018). Prevalence of parent-reported ADHD diagnosis and associated treatment

422 among US children and adolescents, 2016. Journal of Clinical Child \& Adolescent

423 Psychology, 47(2), 199-212.

424 Davis, D. W., Feygin, Y., Creel, L., Williams, P. G., Lohr, W. D., Jones, V. F., Le, J., Pasquenza,

425 N., Ghosal, S., Jawad, K., \& Yan, X. (2019). Longitudinal trends in the diagnosis of

426 attention-deficit/hyperactivity disorder and stimulant use in preschool children on

427 Medicaid. Journal of Pediatrics, 207, 185-191. doi: 10.1016/j.jpeds.2018.10.062

428 Davis, C. S., Piper, B. J., Gertner, A. K., Rotter, J. S. (2020). Opioid prescribing laws are not

429 associated with short-term declines in prescription opioid distribution. Pain Medicine

430 2020;21(3):532-537. doi: 10.1093/pm/pnz159.

431 Dolder, C. R., Davis, N. L., \& McKinsey, J. (2010). Use of psychostimulants in patients with

432 dementia. Annals of Pharmacotherapy, 44(10), 1624-1632. doi: 10.1345/aph.1P341. 
433 Drug Enforcement Administration (2021). Drug Scheduling. Accessed 2/21/2021 at:

434

435

436

437

438

439

440

441

442

443

444

445

446

447

448

449

450

451

452

453

454

455

456

457

458

459

460

461

462

463

464

https://www.dea.gov/drug-scheduling\#: :text=Schedule\%20II\%20drugs\%2C \%20substances $\% 2 C \% 20$ or, drugs\%20are\%20also\%20considered\%20dangerous.

Drug Enforcement Administration, Diversion Control Division, U.S Department of Justice. (2010- 2017). ARCOS Retail Drug Summary Reports. Retrieved from https://www.deadiversion.usdoj.gov/arcos/retail drug summary/.

Epstein, J. N., \& Loren, R. E. (2013). Changes in the definition of ADHD in DSM-5: Subtle but important. Neuropsychiatry, 3(5), 455. doi: 10.2217/npy.13.59

Fulton, B. D., Scheffler, R. M., \& Hinshaw, S. P. (2015). State variation in increased ADHD prevalence: Links to NCLB school accountability and state medication laws. Psychiatric Services, 66(10):1074-1082. doi.org/10.1176/appi.ps.201400145.

Fusar-Poli P, Rubia K, Rossi G, Sartori G, Balottin U. Groenman, A. P., Schweren, L. J. (2012). Striatal dopamine transporter alterations in ADHD: pathophysiology or adaptation to psychostimulants? A meta-analysis. American Journal of Psychiatry 169(3), 264-72. doi: 10.1176/appi.ajp.2011.11060940.

Groenman, A. P., Schweren, L. J., Dietrich, A., \& Hoekstra, P. J. (2017). An update on the safety of psychostimulants for the treatment of attention-deficit/hyperactivity disorder. Expert Opinion on Drug Safety, 16(4), 455-464.

Guerdkikova, A. I., Mori, N., Casuto, L. S., McElroy, S. E. (2016). Novel pharmacologic treatment in acute binge eating disorder - role of lisdexamfetamine. Neuropsychiatric Disease and Treatment, 12, 833-841.

Heo, Y. A., \& Duggan, S. T. (2017). Lisdexamfetamine: A review in binge eating disorder. CNS Drugs, 31(11), 1015-1022. doi: 10.1007/s40263-017-0477-1.

Huber, R. S., Kim, T. S., Kim, N., Kuykendall, M. D., Sherwood, S. N., Renshaw, P. F., \& Kondo, D. G. (2018). Association between altitude and regional variation of ADHD in youth. Journal of Attention Disorders, 22(14), 1299-1306. doi: 10.1177/1087054715577137.

Hulkower, R. L., Kelley, M., Cloud, L. K., Visser, S. N. (2017). Medicaid prior authorization policies for Medication treatment of Attention Deficit/Hyperactivity Disorder in young children, United States, 2015. Public Health Reports 132(6):654-659. doi: $10.1177 / 0033354917735548$.

Ji, X., Druss, B. G., Lally, C., Cummings, J. R. (2018). Racial/ethnic differences in patterns of 
465

466

467

468

469

470

471

472

473

474

475

476

477

478

479

480

481

482

483

484

485

486

487

488

489

490

491

492

493

494

495

496

497

discontinuous medication treatment among Medicaid-insured youth with ADHD.

Psychiatric Services 69(3): 322-331. doi:10.1176.appi.ps.201600469.

Kaiser Family Foundation (2021). Status of state action on the Medicaid expansion decision. Accessed 2/21/2021 at: https://www.kff.org/healthreform/state-indicator/state-activity-around-expanding-medicaid-under-the-affordablecare-act/?activeTab=map\&currentTimeframe=0\&selectedDistributions=status-ofmedicaid-expansion-decision\&selectedRows=\%7B\%22wrapups $\% 22: \% 7 \mathrm{~B} \% 22$ unitedstates\%22:\%7B\%7D\%7D\%7D\&sortModel=\%7B\%22colId\%22:\%22Location \%22,\%22sort $\% 22: \% 22$ asc\%22\%7D

Kandland, M.E., Klein-Schwartz, W. (2015). Comparison of lisdexamfetamine and dextroamphetamine exposures reported to U.S. poison centers. Journal of Clinical Toxicology 53, 477-485. doi: 10.3109/15563650.2015.1027903.

King, S.A., Casavant, M.J., Spiller, H.A., Hodges, N. L., Chounthirath, T, Smith, G. A. (2018). Pediatric ADHD medication exposures reported to US poison control centers. Pediatrics 141, e20173872.

Kollins, S. H., MacDonald, E. K., \& Rush, C. R. Assessing the abuse potential of methylphenidate in nonhuman and human subjects: A review. Pharmacology Biochemistry \& Behavior 2001; 68(3):611-27. doi: 10.1016/s0091-3057(01)00464-6.

Marrast, L., Himmelstein, D. U., \& Woolhandler, S. (2016). Racial and ethnic disparities in mental health care for children and young adults: A national study. International Journal of Health Services, 46(4), 810-824. doi: 10.1177/0020731416662736

National Institute on Drug Abuse; National Institutes of Health; U.S. Department of Health and Human Services. (2018, December). Monitoring the Future 2018 Survey Results. https://d14rmgtrwzf5a.cloudfront.net/sites/default/files/nida-mtf-2018.pdf

Pashmineh, Azar A. R., Cruz-Mullane, A., Podd, J.C., Lam, W.S., Kaleem, S.H., Lockard, L.B., et al. Piper, B. J. (2020). Rise and regional disparities in buprenorphine utilization in the United States. Pharmacoepidemiology \& Drug Safety 2020 Jun;29(6):708-715. doi: 10.1002/pds. 4984

Pennap, D., Burcu, M., Safer, D. J., \& Zito, J. M. (2017). Hispanic residential isolation, ADHD diagnosis and stimulant treatment among Medicaid-insured youth. Ethnicity \& Disease, 27(2), 85. doi: 10.18865/ed.27.2.85

Pew Research Center (2021). Accessed 2/27/2021 at: https://www.pewresearch.org/ Piper, B. J., McCall, K. L., Kogan, L. R., \& Hellyer, P. (2020). Assessment of controlled 
498

499

500

501

502

503

504

505

506

507

508

509

510

511

512

513

514

515

516

517

518

519

520

521

522

523

524

525

526

527

528

529

530

substance distribution to U.S. veterinary teaching institutions from 2006 to 2019.

Frontiers of Veterinary Sciences, 7, 714646. doi: 10.3389/fvets.2020.615646.

Piper, B. J., Ogden, C. L., Simoyan, O. M., Chung, D. Y., Caggiano, J. F., Nichols, S. D., \& McCall, K. L. (2018a). Trends in use of prescription stimulants in the United States and territories, 2006 to 2016. PloS One, 13(11). doi: 10.1371/journal.pone.0206100

Piper, B. J., Shah, D. T., Simoyan, O. M., McCall, K. L., Nichols, S. D. (2018b). Trends in medical use of opioids in the U.S., 2006-2016. American Journal of Preventive Medicine, 2018, 54(5):652-660. doi: 10.1016/j.amepre.2018.01.034.

Punja, S., Shamseer, L., Hartling, L., Urichuk, L., Vandermeer, B., Nikles, J., Vohra S. (2016). Amphetamines for attention deficit hyperactivity disorder (ADHD) in children and adolescents. Cochrane Database of Systematic Reviews. 2016; 2:CD009996. doi: 10.1002/14651858.CD009996.pub2.

Raghavan, R., Brown, D. S., Thompson, H., Ettner, S. L., Clements, L. M., Key, W. (2012). Medicaid expenditures on psychotropic medications for children in the child welfare system. Journal of Child \& Adolescent Psychopharmacology 22(3):182-189. doi: 10.1089/cap.2011.0135.

Rasmussen, N. (2015). Amphetamine-type stimulants: The early history of their medical and non-medical uses. International Review of Neurobiology 120, 9-25. doi: 10.1016/bs.irn.2015.02.001.

Simpson, K. J., Moran, M. T., Foster, M. L., Shah, D. T., Chung, D. Y., Nichols, S. D., McCall K.L., and Piper B.J. (2019). Descriptive, observational study of pharmaceutical and nonpharmaceutical arrests, use, and overdoses in Maine. BMJ Open 9:e027117. doi: 10.1136/ bmjopen-2018-027117.

Stevens, J. R., Wilens, T. E., \& Stern, T. A. (2013). Using stimulants for attention-_ deficit/ hyperactivity disorder: Clinical approaches and challenges. The Primary Care Companion for CNS Disorders, 15(2). doi: 10.4088/PCC.12f01472

Storebø, O. J., Ramstad, E., Krogh, H. B., Nilausen, T. D., Skoog, M., Holmskov, M., Rosendal, S., Groth, C., Magnusson, F.L., Moreira-Maia, C.R. and Gillies, D., (2015). Methylphenidate for children and adolescents with attention deficit hyperactivity disorder (ADHD). Cochrane Database of Systematic Reviews, 11: CD009885. doi: 10.1002/14651858.CD009885.pub2.

Subcommittee on Attention-Deficit/Hyperactivity Disorder \& Steering Committee on Quality Improvement and Management. (2011). ADHD: Clinical practice guideline for the 
531

532

533

534

535

536

537

538

539

540

541

542

543

544

545

546

547

548

549

550

551

552

553

554

555

556

557

558

559

560

561

562

diagnosis, evaluation, and treatment of attention-deficit/hyperactivity disorder in children and adolescents. Journal of the American Academy of Pediatrics, 128(5), 1007-1022. doi: 10.1542/peds.2011-2654

Substance Abuse and Mental Health Services Administration. (2019). Key substance use and mental health indicators in the United States: Results from the 2018 National Survey on Drug Use and Health (HHS Publication No. PEP19-5068, NSDUH Series H-54). Rockville, MD: Center for Behavioral Health Statistics and Quality, Substance Abuse and Mental Health Services Administration. Retrieved from https://www.samhsa.gov/data/ .

Velasco-Mondragon, E., Jimenez, A., Palladino-Davis, A. G., Davis, D., \& Escamilla-Cejudo, J. A. (2016). Hispanic health in the USA: a scoping review of the literature. Public Health Reviews, 37(1), 31. doi: 10.1186/s40985-016-0043-2.

Visser, S. N., Danielson, M. L., Bitsko, R. H., Holbrook, J. R., Kogan, M. D., Ghandour, R. M., Perou, R., \& Blumberg, S. J. (2014). Trends in the parent-report of health care providerdiagnosed and medicated attention-deficit/hyperactivity disorder: United States, 20032011. Journal of the American Academy of Child \& Adolescent Psychiatry, 53(1), 34-46. doi: 10.1016/j.jaac.2013.09.001.

Wang, G. J., Volkow, N. D., Wigal, T., Kollins, S. H., Newcorn, J. H., Telang, F., Logan, J., Jayne, M., Wong, C.T., Han, H., \& Fowler, J.S. (2016). Long-term stimulant treatment affects brain dopamine transporter level in patients with attention deficit hyperactive disorder. PLoS One 8(5):e63023. doi: 10.1371/journal.pone.0063023.

Wei, Y. J. J., Zhu, Y., Liu, W., Bussing, R., \& Winterstein, A. G. (2018). Prevalence of and factors associated with long-term concurrent use of stimulants and opioids among adults with attention-deficit/hyperactivity disorder. JAMA Network Open, 1(4): e181152. doi: 10.1001/jamanetworkopen.2018.1152.

Wineinger, N. E., Zhang, Y., \& Topol, E.J. (2019). Trends in prices of popular brand-name prescription drugs in the United States. JAMA Network Open, 2(5):e194791. doi:10.1001/jamanetworkopen.2019.4791.

Wolraich, M., Brown, L., Brown, R.T., DuPaul, G., Earls, M., Feldman, H. M., Theodore G Ganiats, T. G., Kaplanek, B., Meyer, B., Perrin, J., Pierce, K., Reiff, M., Stein, M. T., Visser, S., American Academy of Pediatrics, Subcommittee on Attention-Deficit/Hyperactivity Disorder; Steering committee on quality improvement and management. (2011). ADHD: clinical practice guideline for the diagnosis, evaluation, 
563 and treatment of attention-deficit/hyperactivity disorder in children and adolescents.

564 Pediatrics, 128(5):1007-1022. doi: 10.1542/peds.2011-2654.

565 Xu, G., Strathearn, L., Liu, B., Yang, B., \& Bao, W. (2018). Twenty-year trends in diagnosed

566 attention-deficit/hyperactivity disorder among US children and adolescents, 1997-2016.

567 JAMA Network Open, 1(4), e181471-e181471. 


\section{Figure Captions}

569 Figure 1. A. Weight per stimulant per state showed a $67.5 \%$ and $76.7 \%$ increase in amphetamine (*paired

570 t-test $\mathrm{p}<0.05$ in comparison to 2010) and lisdexamfetamine (*paired t-test $p<0.05$ in comparison to 2010)

571 and a 3.0\% decrease in methylphenidate (paired t-test $\mathrm{p}=0.8590$ ). B. Average percent change of 50 states

572 data in Daily Dose/Person from 2016-2017 for amphetamine (+4.6\%), lisdexamfetamine (+2.3\%), and

573 methylphenidate (-1.4\%). Lisdexamfetamine was significantly different from amphetamine ( ${ }^{\mathrm{a}} \mathrm{t}$-test $p$

$574<0.05$ ) and methylphenidate ( ${ }^{\mathrm{m}} \mathrm{t}$-test $p<0.05$ ). C. Heat map of United States depicting percent change in

575 total stimulant daily dose/population per state from 2016-2017. D, E, F. Percent change in daily dose and

576 population adjusted analysis per state for amphetamine (D), methylphenidate (E), and lisdexamfetamine

577 (F) reveals $85.0 \%$ of states increased their amphetamine, 72.0\% increased their lisdexamfetamine, and

578 86.0\% states decreased their methylphenidate use. Significant states were marked if $1.96 *$ SD greater or

579 less than the mean for each stimulant. The percent change for New Mexico was excluded as an outlier ( -

580 17.45\% for amphetamine, $-\mathbf{- 1 9 . 9 8 \%}$ for methylphenidate, and -36.63\% for lisdexamfetamine).

581

582 Figure 2. A Heat map for Daily Dose/Population per state for 2017. B Person/Day per Region from

583 2014-2017 indicated a significant difference with the West compared to the South, Midwest and Northeast

584 from 2014-2017 ( *t-test $p<0.05$ ). Time points were slightly offset for display purposes.

585

586 Figure 3. Negative correlations between total Daily Dose/Population value and the percent Hispanic per

587 state for 2016. (A, amphetamine: $r(48)=-0.43, p=0.0017$; B, methylphenidate: $r(48)=-0.64, p<0.0001$;

588 C, lisdexamfetamine: $r(48)=-0.49, p \leq 0.0001)$. Negative correlations between total Daily

589 Dose/Population value and percent Hispanic per state for 2017 (D. amphetamine $r(48)=-0.43, p<0.005$;

590 E, methylphenidate: $r(48)=-0.65, p<0.0001$; F, lisdexamfetamine $r(48)=-0.52, p \leq 0.0001$ ).

591

592

593

594 Table Caption

Peer] reviewing PDF | (2020:10:53393:2:1:NEW 3 Nov 2021) 
595

596 Table 1. Percent Medicaid amount reimbursed and number of prescriptions per stimulant (A). Percent

597 Medicaid amount reimbursed and number of prescriptions by formulation for methylphenidate (B),

598 amphetamine (C) and atomoxetine (D).

599

600

601

602

603

604

605

606

607

608

609

610

611

612

613

614

615

616

617

618

619

620

621

622

623

624

625

626

Table 1. Percent of Medicaid amount reimbursed and number of prescriptions per stimulant (A) and by formulation for methylphenidate (B), amphetamine (C) and atomoxetine (D).

A. Total

\% Amount Reimbursed \% Number of Prescriptions 
627

628

629

630

631

632

633

634

635

636

637

638

639

640

641

642

643

644

645

646

647

648

649

650

651

652

653

654

655
51.67

25.97

18.42

3.95

B. Methylphenidate

$$
\text { methylphenidate }
$$

Focalin

Concerta

dexmethylphenidate

Quillichew

Quillivant

Aptensio X

Daytrana

Cotempla X

other

C. Amphetamine

Adderall

mixed amphetamine

dextroamphetamine

Adzenys

Dyanavel X

Mydayis

Evekeo

Procentra

Other

D. Atomoxetine

atomoxetine

Strattera
48.00

25.81

11.61

6.39

3.26

1.45

1.13

1.05

0.83

0.45

51.46

20.89

20.32

2.52

2.44

0.90

0.67

0.50

0.30

75.17

24.83
45.36

33.62

16.27

4.75

62.51

14.16

6.11

12.18

1.86

0.79

0.91

0.62

$<.01$

$<.01$

21.54

24.62

51.59

0.77

0.79

$<.01$

$<.01$

$<.01$

$<.01$

91.49

8.50 


\section{Figure 1}

Weight per stimulant per state

Figure 1. A. Weight per stimulant per state showed a $67.5 \%$ and $76.7 \%$ increase in amphetamine (*paired t-test $p<0.05$ in comparison to 2010 ) and lisdexamfetamine (*paired t-test $p<0.05$ in comparison to 2010 ) and a 3.0\% decrease in methylphenidate (paired t-test $p=0.8590)$. B. Average percent change of 50 states data in Daily Dose/Person from 2016-2017 for amphetamine (+4.6\%), lisdexamfetamine $(+2.3 \%)$, and methylphenidate (-1.4\%). Lisdexamfetamine was significantly different from amphetamine ( ${ }^{\mathrm{a}}$-test $\left.p<0.05\right)$ and methylphenidate ( ${ }^{\mathrm{m}} \mathrm{t}$-test $p<0.05$ ). C. Heat map of United States depicting percent change in total stimulant daily dose/population per state from 2016-2017. D, E, F. Percent change in daily dose and population adjusted analysis per state for amphetamine (D), methylphenidate (E), and lisdexamfetamine $(\mathbf{F})$ reveals $85.0 \%$ of states increased their amphetamine, $72.0 \%$ increased their lisdexamfetamine, and $86.0 \%$ states decreased their methylphenidate use. Significant states were marked if 1.96*SD greater or less than the mean for each stimulant. The percent change for New Mexico was excluded as an outlier ( $-17.45 \%$ for amphetamine, $-19.98 \%$ for methylphenidate, and $-36.63 \%$ for lisdexamfetamine). 
A

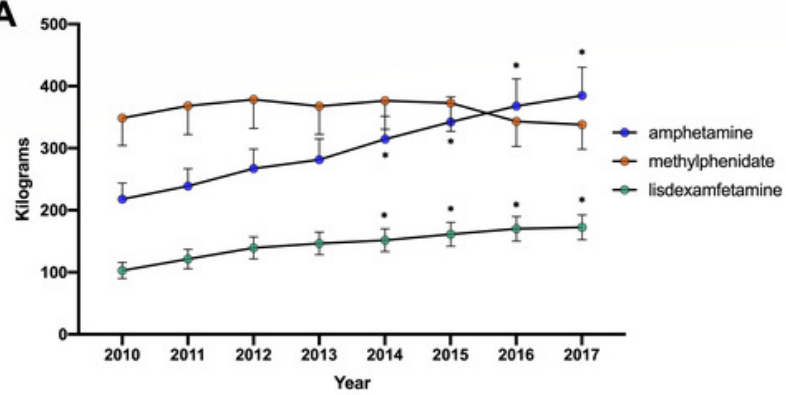

B

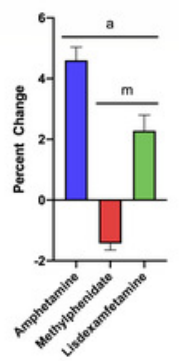

E $\begin{gathered}\text { Percent Change in Methylphenidate } \\ \text { Daily Dose/Population from 2016-2017 }\end{gathered}$

D

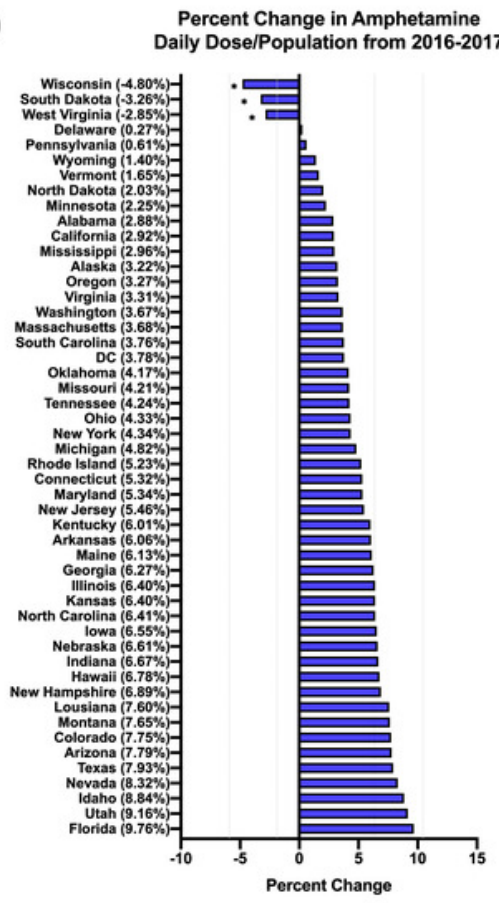

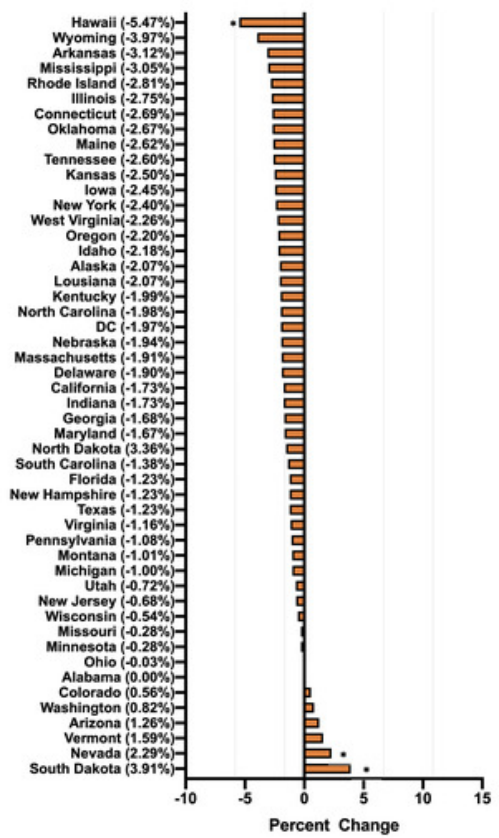

C

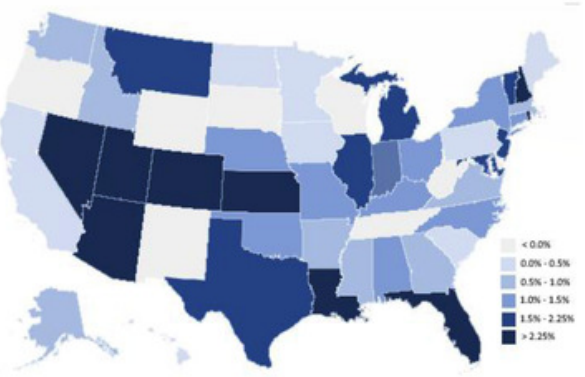

F $\quad \begin{aligned} & \text { Percent Change in Lisdexamfetamine } \\ & \text { Daily Dose/Population from 2016-2017 }\end{aligned}$

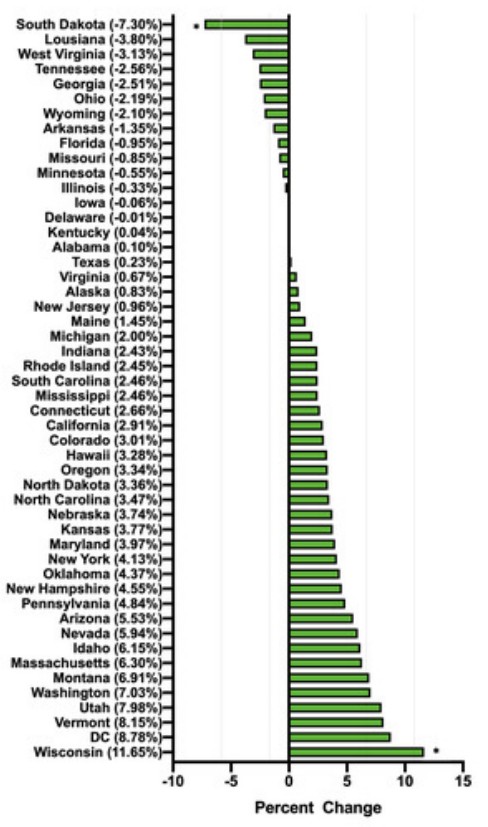


Figure 2

Heat map and regional analysis.

Figure 2. A Heat map for Daily Dose/Population per state for 2017. B Person /Day per Region from 2014-2017 indicated a significant difference with the West compared to the South, Midwest and Northeast from 2014-2017 ( $*$ t-test $p<0.05$ ). Time points were slightly offset for display purposes.

A

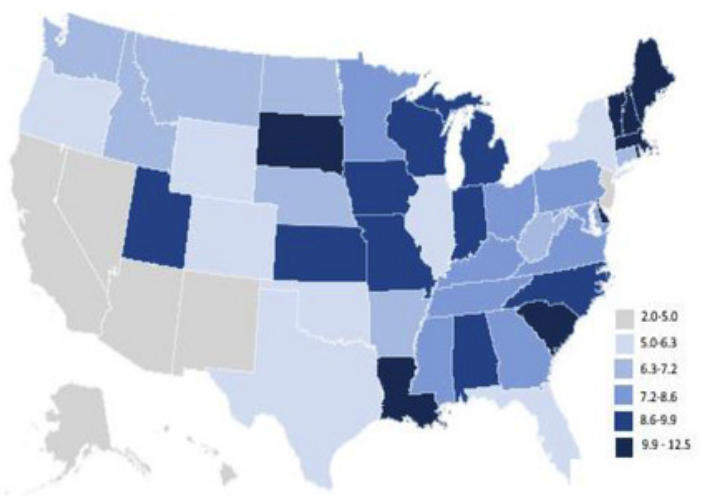

B

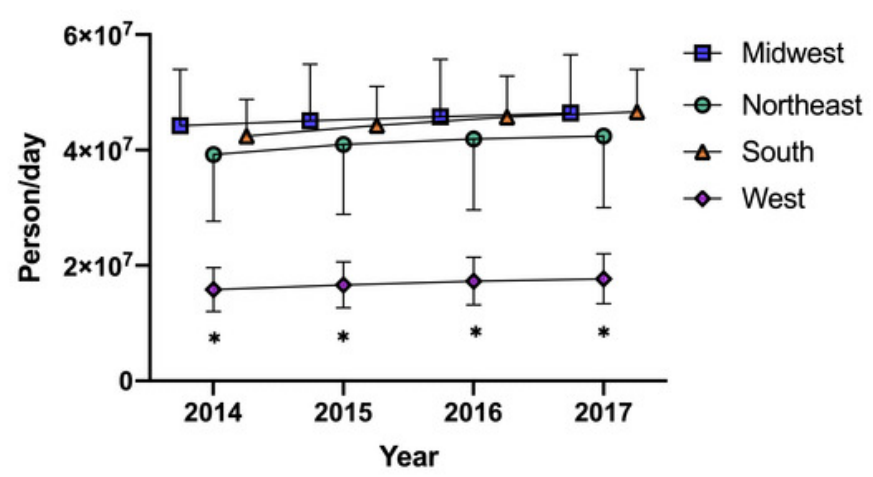


Figure 3

Scatterplots and negative correlations

Figure 3. Negative correlations between total Daily Dose/Population value and the percent Hispanic per state for 2016. (A, amphetamine: $r(48)=-0.43, p=0.0017$; B, methylphenidate: $r(48)=-0.64, p<0.0001 ; \mathbf{C}$, lisdexamfetamine: $r(48)=-0.49, p<$ 0.0001). Negative correlations between total Daily Dose/Population value and percent Hispanic per state for 2017 (D. amphetamine $r(48)=-0.43, p<0.005$; E, methylphenidate: $r(48)=-0.65, p<0.0001 ; \mathbf{F}$, lisdexamfetamine $r(48)=-0.52, p<0.0001)$.

A

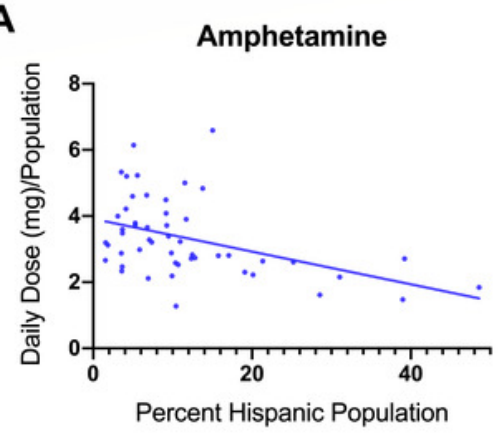

D

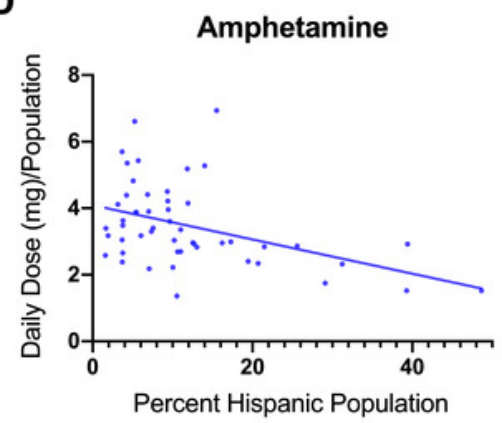

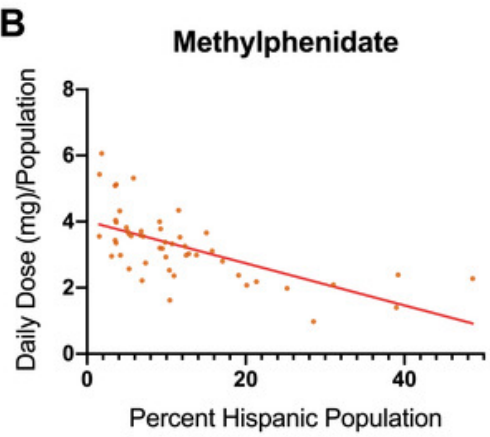

E

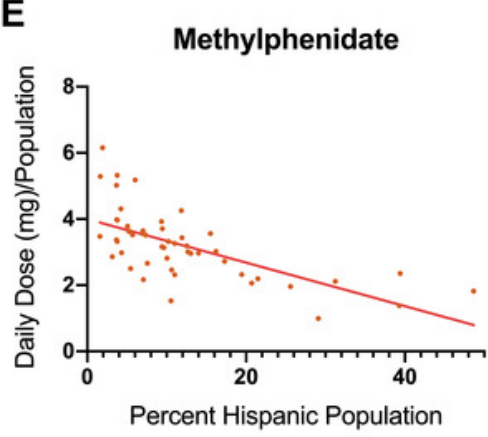

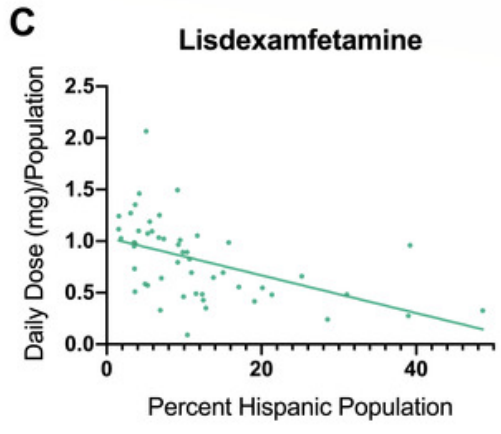

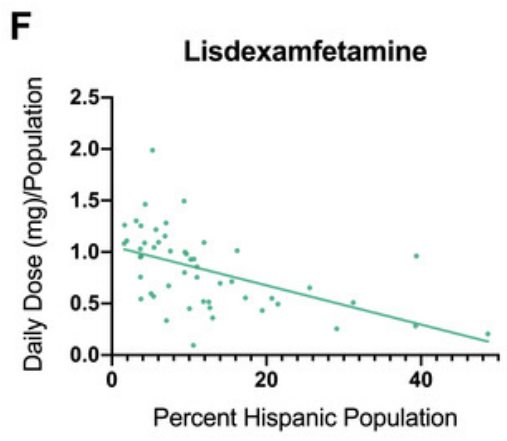

